\title{
Numerically Exact Approach to Ultra-Small Particles in Near-Critical Mixtures
}

\author{
A. DRZEWIŃSKI* \\ Institute of Physics, University of Zielona Góra, Prof. Z. Szafrana 4a, 65-516 Zielona Góra, Poland \\ (Received November 8, 2018; in final form January 22, 2019)

\begin{abstract}
The critical Casimir forces between solute particles in a solvent can arise from the medium fluctuations. The properties of ultra-small particles immersed in a binary liquid mixture close to its consolute point $T_{c}$ are studied using the exact statistical-mechanical derivation based on the two-dimensional Ising model. Each solute particle is represented by a local magnetic field acting on an individual spin. The ensemble average for the distance between particles placed on the torus is determined as a temperature-dependent function. It has been shown how the presence of other particles modifies the separation distance for a pair of particles with the same or opposing preferential adsorption.
\end{abstract}

DOI: 10.12693/APhysPolA.135.520

PACS/topics: 75.10.Hk, 05.20.Jj, 05.40.-a

\section{Introduction}

The first direct measurement of critical Casimir force $[1,2]$ between a single colloidal sphere and a flat silica surface in a water-2,6-lutidine mixture was performed ten years ago [3]. It created a tremendous impulse for further experimental and theoretical studies of the critical behaviour of colloidal suspensions [4-11]. One of the most intensively researched issues is the aggregation transition originating from the confinement of the solvent critical fluctuations between the surfaces of distinct colloids $[3,5,12]$. Such problems are often analysed using mean-field theories or simulations [13-17]. Nevertheless, there is still much to understand, particularly near the critical point where the role of fluctuations is enhanced and the accuracy of these methods is limited.

A binary mixture of liquids A and B can exist as two separate phases or as a single phase, depending on the temperature. The temperature-composition diagram of the A-B mixture can have an upper critical solution temperature or a lower one or both of them. In the present paper, this first case will be mostly referred, where the $T>T_{c}$ regime corresponds to the mixed (disordered) state of the binary mixture, whereas $T<T_{c}$ relates to the demixed (ordered) state. By tuning temperature, the solvent correlation length can be changed, affecting the magnitude and range of interactions. The nature of the observed critical behavior significantly depends on the surface properties of solute particles [18]. Generally, these forces are attractive if the boundary conditions are symmetric, and repulsive if the boundary conditions are asymmetric [2].

Typically, the size involving the solute particles is characteristic for a mesoscopic system (a few hundred nm). However, when we deal with ultra-small particles, their

*e-mail: a.drzewinski@if.uz.zgora.pl internal structure can be neglected. The only thing that matters is the fact that their surface may prefer one of the two components of the mixture. In fact, this approach is focused on a very small amount of solute particles of a size comparable with the size of the solvent particles. Taking the system of water and 2,6-lutidine with a molecular diameter of $0.275 \mathrm{~nm}$ and $0.67 \mathrm{~nm}$ [19] as a binary mixture, only the ultra-small particles with a size of a few $\mathrm{nm}$ could be used $[20,21]$. The interactions between solute particles embedded in a fluctuating medium are expected when the two particles are separated by a distance shorter than the correlation length of local concentration fluctuations. As the correlation length diverges when the critical temperature is approached, the most pronounced effects are found here. Moreover, because of the universality of critical phenomena, they are rather insensitive to the specific materials, so one can expect similar temperature dependence for various solvents.

For a classical binary mixture, the consolute (demixing) point corresponds to the second-order phase transition of the Ising universality class [22]. Therefore, the system properties have been modelled by the twodimensional Ising model on the square lattice on a torus with a linear size up to $N=15$. Any individual spin represents a small volume of the binary mixture (a cell). The states of spin "up"/"down" refer to the cell with a high/low concentration of the component, e.g. A, respectively. Thanks to the choice of the torus geometry, the influence of the walls has been eliminated. Nevertheless, as the system has a finite size, the critical point position is shifted from the critical point position in the thermodynamic limit [23] (for a square lattice, $T_{c} \approx 2.269 \mathrm{~J} / k_{B}$ ). It is worth adding that generally for periodic systems, there are much weaker corrections to scaling than for the system where the space translation symmetry is broken.

An exact expression of the partition function for the Ising system on the square lattice wrapped on a torus was given by Kaufman [24]. The exact partition function of the Ising model on the general two-dimensional lattice on 
a torus was derived by Morita [25]. In the present paper, the key is to derive the partition function for the Ising system (the dispersion medium) with a non-zero magnetic field at a few lattice sites. The sign of the magnetic field corresponds to the affinity of a solute particle, that prefers to be surrounded only by one component of the mixture. Based on the equilibrium statistical thermodynamics, the statistics of configurations is governed by a Boltzmann weight that depends both on the position of the solute particles and on the state of the medium. Therefore, the temperature plays the role of a unique parameter to control the range and strength of interaction. In order to determine the average distance between individual solute particles, the canonical partition function of the whole system should be used. It contains all the information needed to predict the macroscopic behavior of a system. The study results indicate that despite the simplicity of the model, it seems to capture the essential physics of a few ultra-small particles in a binary mixture over the wide range of temperatures.

This paper has two objectives. First, we intend to check whether the ultra-small particles interacting by the Casimir-like forces reproduce the main characteristics of the critical Casimir forces for two particles with various preferential adsorptions. Second, as the experiments provide an evidence for non-additive nature of the critical Casimir forces [7, 26], the interactions cannot be described with pair potentials in this case. As the proposed numerically exact method can capture such collective behaviour, we intend to investigate how the presence of an extra particle modifies the average distance between the other two particles.

The remainder of this paper is organized as follows. In Sect. 2 the model system is outlined, whereas the results of the investigations are presented in Sect. 3. Finally, in Sect. 4, some concluding remarks are made.

\section{Model}

The parameter that is crucial to describe the phenomenon of solute particle aggregation is the average distance between particles. However, when all nonequivalent particle settings on the torus and, for each setting, all spin configurations should be taken into account, therefore, the number of system states becomes very large. Fortunately, these requirements can be met by the transfer matrix method [27, 28] that is powerful and can be applied to the Ising-like systems, also with the magnetic field acting on individual sites of the lattice.

The two-dimensional Ising model, on the square lattice $N \times N$ with cyclic boundary conditions in the both directions, is considered. The energy for a configuration $\{S\}$ of spins is given by the following Hamiltonian:

$$
\mathcal{H}=-J\left(\sum_{\{i, j\},\{k, l\}} S_{i, j} S_{k, l}-h_{1,2} \sum_{\begin{array}{c}
\text { particle } \\
\text { positions }
\end{array}} S_{i, j}\right),
$$

with $J>0$ and $S_{i, j}= \pm 1$. The first sum is taken over the nearest neighbors, while the second one is performed on the individual spins only. Affecting the medium in the immediate vicinity the presence of solute particles is manifested only by favouring one of the mixture phases. For different solute particles, the preferential adsorption can vary, which entails the presence of different on-site magnetic fields $h_{1}$ or $h_{2}$. Moreover, assuming that the system is at the critical concentration, a bulk field conjugated to the order parameter takes the value zero.

For the torus of linear size $N$, the average distance between any two lattice sites can be determined as the arithmetic average for all site positions giving the (approximate) formula $L=0.38078 N+0.04092$. Therefore, bearing in mind the previous paragraph, one can say that the size $L$ corresponds to the average distance between two colloidal particles without preferential adsorption. These sizes are listed in Table I.

Characteristic distances on a torus.

TABLE I

\begin{tabular}{c|c}
\hline \hline $\begin{array}{c}\text { Linear } \\
\text { torus size } N\end{array}$ & $\begin{array}{c}\text { Average } \\
\text { distance } L\end{array}$ \\
\hline 7 & 2.707756 \\
9 & 3.467021 \\
11 & 4.228267 \\
13 & 4.990644 \\
15 & 5.753726
\end{tabular}

In the Ising model, the deviation from the criticality is described by the reduced temperature $\tau=\left(T-T_{c}\right) / T_{c}$, where $T_{c}$ is the bulk critical temperature [29]. The appropriate scaling variable related to temperature is the ratio between the average distance and bulk correlation length in the mixed phase $z=\operatorname{sgn}(\tau) L / \xi_{\tau}=L \tau^{\nu} / \xi_{0}^{+}$. For the two-dimensional Ising model, the universal exponent is $\nu=1$ and non-universal amplitude takes on value $\xi_{0}^{+}=0.5673[30,31]$.

In order to calculate the probability that the two solute particles are at the distance $l_{i}$, the following formula can be applied:

$$
p_{i}=\sum_{\begin{array}{c}
\text { all spin configurations } \\
\text { particles at the distance } l_{i}
\end{array}} \mathrm{e}^{-\beta \mathcal{H}\left(\{S\}, h_{1,2}\right)} / \mathcal{Z},
$$

where $\beta=1 / k_{\mathrm{B}} T, k_{\mathrm{B}}$ is the Boltzmann constant and $\mathcal{Z}$ is the partition function

$$
\mathcal{Z}=\sum_{\substack{\text { all system } \\ \text { states }}} \mathrm{e}^{-\beta \mathcal{H}\left(\{S\}, h_{1,2}\right)} .
$$

Now, averages of physical quantities, as the separation distance of two solute particles, can be expressed by the formula

$$
\langle l\rangle=\sum_{\text {all distances }} p_{i} l_{i} .
$$

As it does not limit the considerations, one of the solute particles has always been located in the same lattice site. 
Furthermore, using the symmetry of the square lattice on a torus, the number of different positions of the second particle can be significantly reduced, which greatly simplifies the calculations. When three particles are present and the distance between a given pair of particles is to be determined, all non-equivalent positions of the third particle has to be taken into account.

\section{Results}

The transfer matrix method, allows to study the impact of temperature on the ensemble average distance in any pair of solute particles. In general, the method is applicable whenever the partition function can be expressed as a product of matrices [32]:

$$
\mathcal{Z}=\sum_{\begin{array}{c}
\text { all states of } \\
\text { the 1st column }
\end{array}} \sum_{\begin{array}{c}
\text { all states of } \\
\text { the 2nd column }
\end{array}} \ldots \sum_{\begin{array}{c}
\text { all states of } \\
\text { the N-th column }
\end{array}}\left\langle S_{1}\left|T_{12}\right| S_{2}\right\rangle\left\langle S_{2}\left|T_{23}\right| S_{3}\right\rangle \ldots\left\langle S_{N}\left|T_{N 1}\right| S_{1}\right\rangle \text {. }
$$

For our case, $N$ is the linear size of the torus and thus the number of columns. Because each column consists of $N$ spins, the number of states in each column is $2^{N}$.

When the system is homogeneous, one has to deal with a single execution of repeated multiplication of the same matrix $T \equiv T_{i+1}$. As the model gets complex (for example, due to an on-site magnetic field present in one of the columns), the transfer matrix method also gets complex. Similarly, the nonmagnetic site (the lack of a spin) can be included. As a result, one is forced to execute for all nonequivalent particle positions the multiplication of several types of $T_{i} i+1$ matrices, which significantly increases the computation time.

\subsection{Two particles}

The calculations were carried out in a wide range of magnetic fields for particles with the same or opposing preferential adsorption (see Fig. 1). The results for the representative case $\left|h_{1}\right|=1$ are presented in Figs. 2 and 3. The average distance between particles without preferential adsorption is taken as the reference case.

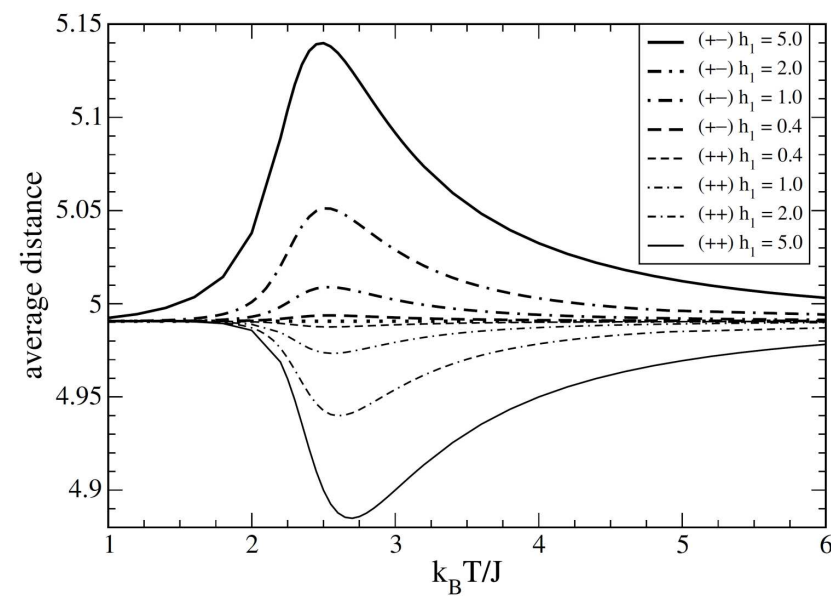

Fig. 1. The temperature-dependent average distance between two solute particles for different preferential adsorptions (the on-site magnetic field). The torus linear size is $N=13$.

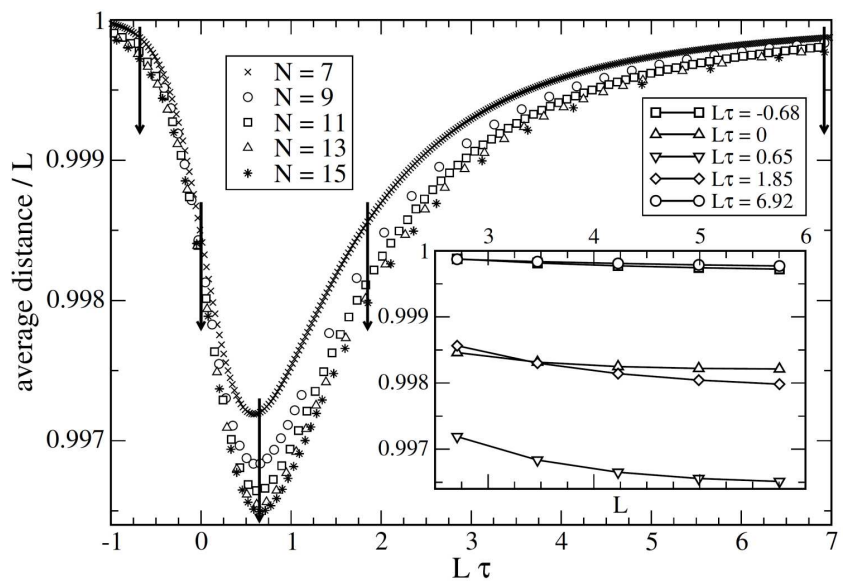

Fig. 2. The normalized average distance between two particles with the same preferential adsorption $\left(h_{1}=1\right)$ as a function of scaling variable for different system sizes. The inset presents the size-dependent average distances for a few values of the scaling parameter $L \tau$ indicated by the arrows in the main panel.

As one could expect, at equilibrium the particles preferring the same component of the mixture are on average closer to each other than the particles without preferential adsorption. Furthermore, a pair of particles preferring different components of the mixture are on average further apart than in the reference case. As the fluctuation-induced forces are the strongest near the continuous phase transition, the effect on changing the distance between particles is the most visible here. Naturally, it can be noticed that even in this case the relative deviation from the reference value $L$ varies by no more than half a percent. The reason lies in the model assumption: the influence of an ultra-small particle is reduced to a single lattice site representing one cell of the binary mixture.

The simplest extension of the point-like particles is to present a solute particle as a single non-magnetic lattice site not interacting with the four nearest neighbors. Moreover, its influence manifests as the magnetic field (a preferential adsorption) acting on all (eight) sites surrounding such a "silent" site (the one-site block). When the identical/opposing magnetic fields from two close- 


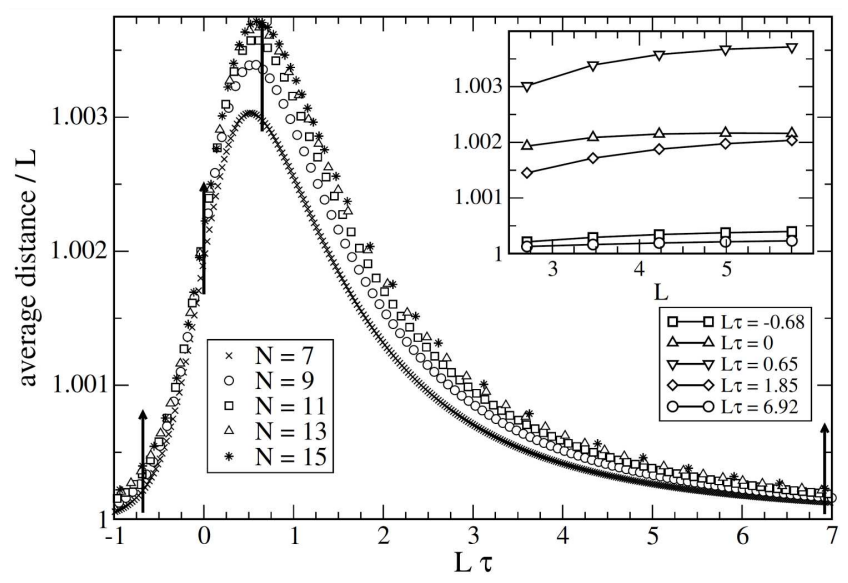

Fig. 3. The normalized average distance between two solute particles with the opposing preferential adsorption $\left(\left|h_{1}\right|=1\right)$ as a function of scaling variable for different system sizes. The inset presents the size-dependent average distances for a few values of the scaling parameter $L \tau$ indicated by the arrows in the main panel.

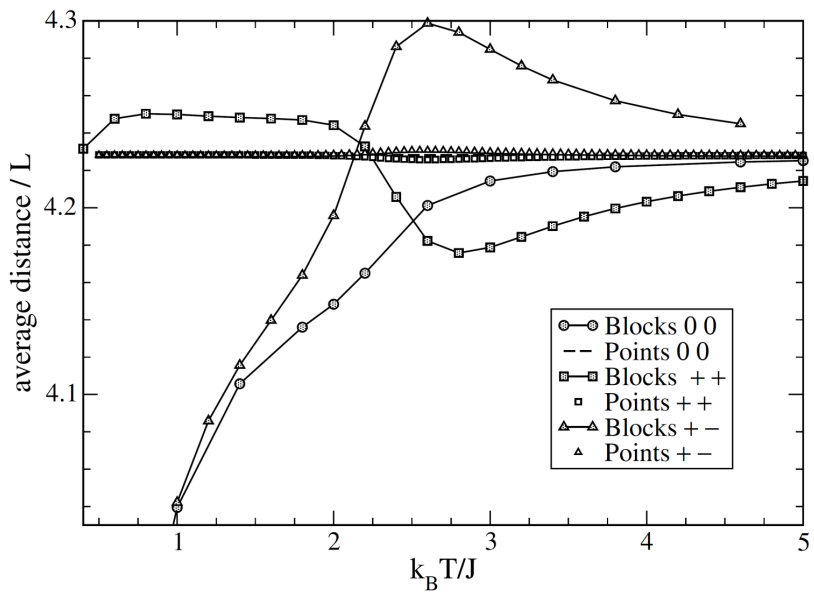

Fig. 4. The comparison of the point-like and one-site block cases for the system size $N=11$ and on-site magnetic field $\left|h_{1}\right|=0,1$.

lying particles overlap, the field value doubles/cancels at the sites belonging to the both surroundings. The comparison of the point-like and one-site block cases for the same system size and the same field has been presented in Fig. 4. As one can see, the effect is clearly stronger when a solute particle is modelled by the one-site block.

For the case without preferential adsorption $\left(h_{1}=\right.$ $0)$, contrary to the point-like particles, when the average distance does not depend on temperature, for the one-block particles, the lower temperature, the smaller average distance between particles. The reason lies in the fact that when the both particles (the "silent" sites) are directly adjacent to each other, the total number of eliminated bonds falls from eight to seven. As a result, for such configurations the total energy decreases by the one-bond energy. The effect is enhanced at low temperatures, when the spins tend to set up in the same direction (the one-bond energy $\approx-J$ ). Therefore, the configurations with two particles lying next to each other become more likely and, as a result, the average distance between the particles decreases. When a preferential adsorption appears $\left(h_{1}>0\right)$ around the both "silent" points, then from a certain value of $h_{1}$ its impact becomes dominant. As a result, configurations with non-adjoining particles are more likely, so the average distance between particles increases. For opposing preferential adsorptions, their influence compensates each other, and thus the average distance between particles does not change significantly for low temperatures. In general, the one-block case was not examined in detail as it is no longer covered by the scope of this article.

Unfortunately, for the point-like case the calculation for systems larger than $N=15$ are very complex and time consuming. Therefore, the limiting form of the curve when $N$ grows cannot be determined conclusively. However, when the system size raises the total number of sites increases, whereas the number of solute particles is fixed. Therefore, their impact on the behavior of the entire system should disappear. Thus, the curves describing the separation distances between particles in pairs, from a certain system size $L_{0}$, should start to decrease. The plots presented as the insets in Figs. 2 and 3 seem to support this scenario. Although, the maxima are not yet visible on any of the drawings, on closer inspection, it can be found that there is a maximum at $L_{0}=4.99 \ldots$ for a pair with opposing preferential adsorptions at $\tau=0$.

\subsection{Three particles}

As it was already emphasized, when three particles are present the pairwise description fails [33]. Therefore, the goal is to determine the average distance between two particles when the third one is present. As it turned out, the key is whether both particles have the same or opposing surface properties. The preferential adsorption of the coupled particles is governed by the parameter $h_{1}$, while the extra particle properties are controlled by the parameter $h_{2}$.

As Fig. 5 shows when both particles have the same surface preferences, the presence of another (identical) particle keeps them somewhat away from each other. This effect seems to be contrary to intuition. Two particles with the same preferential adsorption attract each other due to the medium fluctuations, but when one more identical particle is added, the attraction becomes slightly weaker! This behavior will become clearer when one notices that the third particle is not solely located between the two particles constituting the pair. In order to calculate the equilibrium distance, all allowed positions of the third particle have to be taken into consideration. For the majority of configurations, the additional particle is not located between the particles belonging to the pair, but outside them. As a result, the distance between any two particles in a system of many identical particles will be slightly larger than for an isolated pair. 


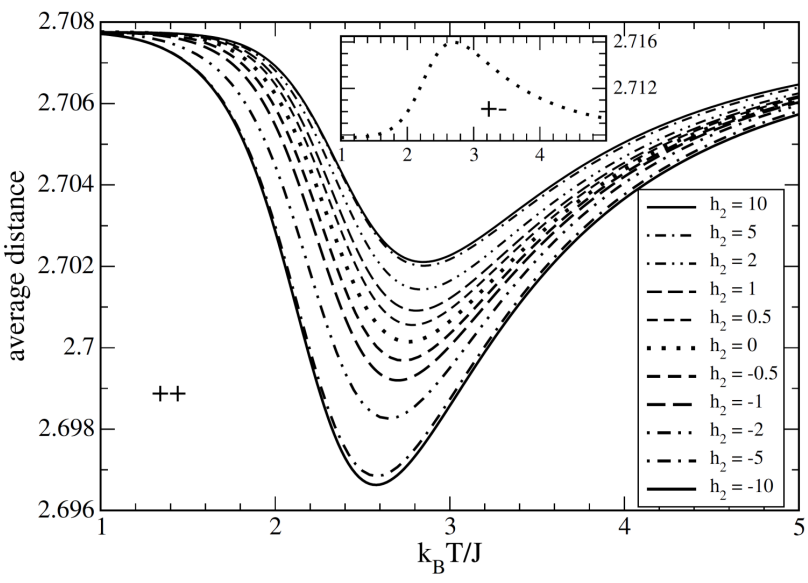

Fig. 5. The temperature-dependent average distance between two identical solute particles $\left(h_{1}=1\right)$ when a three-particle system is considered. Surface properties of the third particle change over a wide range of $h_{2}$ and curves are ordered as in the legend from top to bottom. Inset: the temperature-dependent average distance between two solute particles with opposing preferential adsorptions is insensitive to the presence of an additional particle (regardless of its preferential adsorption). The torus linear size is $N=7$.

In turn when the preferential adsorption of the third particle is opposing to the preferential adsorption in a pair, the final effect is opposite. It leads to a reduction in the distance between particles. Of course, as in the previous case, the dominant behavior between the particles in a selected pair remains unchanged: particles with the same preferential absorption attract each other.

When a couple of particles with opposing surface preferences is considered, the situation is completely different. The presence of other particles about any preferences does not affect the average distance between particles in the pair (see the inset in Fig. 5). The dotted-line curve in the inset symbolizes all curves for $h_{1}= \pm 1$ and any $h_{2}$.

\section{Conclusions}

Suspensions in liquids are of particular interest because of their potential scientific significance and technological applications [34-36]. We have investigated the simple but numerically exactly solvable model for a few solute particles inserted into a binary mixture. The transfer matrix method enables to study the static, equilibrium properties of particles with the same or different adsorption preferences. The degrees of freedom of a few solute particles and solvent have been fully taken into account without any approximation.

In the proposed model the dispersion medium is represented by the Ising model, whereas a solute particle is revealed as an on-site magnetic field. In order to place the proposed model in a broader context, its simplest generalization for a one-block case has been initially analysed, when a solute particle is presented as a single "silent" site not interacting with the nearest neighbors but its influence on the medium is manifested by the preferential adsorption (an on-site magnetic field) of the eight surrounding sites.

In order to obtain the desired functional properties the particle surface is often functionalized with surfactants or macromolecules to induce repulsions or attraction between particles. The present study has confirmed that the critical Casimir-like force between two identical bodies are always attractive and become repulsive if their preferential adsorptions are different. These fluctuationinduced forces are most pronounced near criticality.

Moreover, it has been demonstrated that the average distance between two solute particles with fixed preferential adsorption can be modified if other particles are present. Namely, if two particles immersed in a binary mixture, prefer the same mixture component they still attract each other but this effect can be a bit tuned.

In contrast, solute particles with the opposing preferential adsorption do not change their average distances in the presence of other particles. Therefore, it can be anticipated that the critical aggregation phenomenon is more subtle and dependent on the many-body interactions of colloidal particles with the same and opposing preferences.

\section{Acknowledgments}

The author acknowledges the financial support from the program of the Polish Minister of Science and Higher Education under the name "Regional Initiative of Excellence" in 2019-2022, project no. 003/RID/2018/19, funding amount 11936 596.10 PLN.

\section{References}

[1] M.E. Fisher, P.G. de Gennes, C.R. Sci. Acad. Sci. Ser. B 287, 207 (1978).

[2] S. Rafai, D. Bonn, J. Meunier, Physica A 386, 31 (2007).

[3] C. Hertlein, L. Helden, A. Gambassi, S. Dietrich, C. Bechinger, Nature 451, 172 (2008).

[4] O. Zvyagolskaya, A.J. Archer, C. Bechinger, Europ. Phys. Lett. 96, 28005 (2011).

[5] V.D. Nguyen, S. Faber, Z. Hu, G.H. Wegdam, P. Schall, Nat. Commun. 4, 1584 (2013).

[6] P. Nowakowski, M. Napiórkowski, J. Chem. Phys. 14, 064704 (2014).

[7] C.A.S. Batista, R.G. Larson, N.A. Kotov, Science 350, 1242477 (2015).

[8] A. Ciach, Adv. Biomembr. Lipid Self-Assembly 23, 61 (2016).

[9] S.G. Stuij, M.L. Laurent, T.E. Kodger, A. Maciołek, P. Schall, Soft Matter 13, 5233 (2017).

[10] S.D. Mostovoy, O.V. Pavlovsky, Phys. Lett. A 382, 276 (2018).

[11] O.A. Vasilyev, S. Dietrich, S. Kondrat, Soft Matter 14, 586 (2018). 
[12] R.R. Netz, Phys. Rev. Lett. 76, 3646 (1996).

[13] T.F. Mohry, A. Maciołek, S. Dietrich, J. Chem. Phys. 136, 224903 (2012).

[14] R. Okamoto, A. Onuki, Phys. Rev. E 88, 022309 (2013).

[15] T.F. Mohry, S. Kondrat, A. Maciołek, S. Dietrich, Soft Matter 10, 5510 (2014).

[16] J.R. Edison, N. Tasios, S. Belli, R. Evans, R.V. Roij, M. Dijkstra, Phys. Rev. Lett. 114, 038301 (2015).

[17] J. Benet, F. Paillusson, H. Kusumaatmaja, Phys. Chem. Chem. Phys. 19, 24188 (2017).

[18] C.E. Bertrand, P.D. Godfrin, Y. Liu, J. Chem. Phys. 143, 084704 (2015).

[19] F.T. Starzyk, I. Stan, S. Abelló, A. Bonilla, K. Thomas, C. Fernandez, J.P. Gilson, J.P. Ramirez, J. Catal. 264, 11 (2009).

[20] X. Miao, S.L. Ho, T. Tegafaw, H. Cha, Y. Chang, In Taek Oh, A.M. Yaseen, S. Marasini, A. Ghazanfari, H. Yue, K.S. Chaec, G.H. Lee, RSC Adv. 8, 3189 (2018).

[21] M. Marć, M.R. Dudek, J.J. Kozioł, B. Zapotoczny, Nano 13, 1850142 (2018).

[22] A. Pelissetto, E. Vicari, Phys. Rep. 368, 549 (2002).

[23] K. Binder, Annu. Rev. Mater. Res. 38, 123 (2008).

[24] B. Kaufman, Phys. Rev. 76, 1232 (1949).
[25] T. Morita, J. Phys. A Math. Gen. 19, L1191 (1986).

[26] S. Paladugu, A. Callegari, Y. Tuna, L. Barth, S. Dietrich, A. Gambassi, G. Volpe, Nat. Commun. 7, 11403 (2016).

[27] R.J. Baxter, Exactly Solved Models in Statistical Mechanics, 3rd ed., Academic Press, 1989.

[28] G. Mussardo, Statistical Field Theory: An Introduction to Exactly Solved Models in Statistical Physics, OUP, Oxford 2009.

[29] M. Zubaszewska, A. Maciołek, A. Drzewiński, Phys. Rev. E 88, 052129 (2013)

[30] H.B. Tarko, M.E. Fischer, Phys. Rev. B 11, 1217 (1975).

[31] A. Drzewiński, A. Maciołek, A. Ciach, Phys. Rev. E 61, 5009 (2000).

[32] P. Nowakowski, A. Maciołek, S. Dietrich, J. Phys. A 49, 485001 (2016).

[33] T.G. Mattos, L. Harnau, S. Dietrich, Phys. Rev. E 91, 042304 (2015)

[34] T. Bollhorst, K. Rezwan, M. Maas, Chem. Soc. Rev. 46, 2091 (2017).

[35] I.A. Martínez, C. Devailly, A. Petrosyan, S. Ciliberto, Entropy 19, 77 (2017).

[36] N. Koumakis, C. Devailly, W.C.K. Poon, Phys. Rev. E 97, 062604 (2018). 